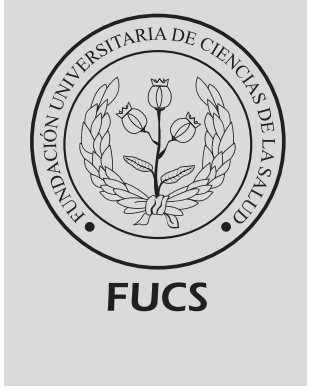

\title{
Análisis de los resultados del contagio del COVID-19 respecto a su distribución geográfica en Colombia
}

\section{Análysis of the geographic distribution of COVID-19 cases in Colombia}

a Ingeniero. Magister en Gestión de la Tecnología Educativa, Especialista en Administración de la Informática Educativa.

Docente de matemáticas e Investigador, Secretaría de Educación de Soacha, Cundinamarca.

\section{R E S U M E N}

Introducción: al cumplirse cien días del primer contagio por coronavirus COVID-19 en Colombia, se realizó un estudio de investigación de los resultados de la enfermedad por su distribución por departamentos y distritos en el país. Objetivo: comparar si hay diferencias en los promedios de personas con contagio positivo, fallecidos y recuperados entre los diferentes departamentos y distritos en Colombia. Materiales y métodos: se utilizó la base de datos de las personas infectadas por el COVID-19 con la información acumulada a 13 de junio de 2020. Se realizó a partir del análisis estadístico de los datos aplicando la prueba T Student para una muestra, se utilizó el paquete estadístico SPSS v. 25. Resultados: los diferentes p-valor de las pruebas, para la variable casos positivos es igual a 0,492, para la variable casos recuperados es igual a 0,897, para la variable fallecidos es igual a 0,819, en los tres casos es mayor a $a=0.05$, de esta manera se acepta Ho, es decir la prueba $\mathrm{T}$ Student para una muestra nos indica que no hay diferencias significativas entre el puntaje de la población y el puntaje obtenido para la muestra por COVID-19. Conclusiones: se presenta mayor población de contagio en la zona Andina de Colombia debido a la mayor densidad de población en esta región, y lo contrario en las regiones de la Amazonía y Orinoquía. Además, se mantiene la misma relación por zonas geográficas en cuanto a personas recuperadas y fallecidas.

Palabras clave: coronavirus, transmisión de enfermedad infecciosa; pandemia.

(C) 2020 Fundación Universitaria de Ciencias de la Salud - FUCS. Este es un artículo Open Access bajo la licencia CC BY-NC-ND (http://creativecommons.org/licenses/by-nc-nd/4.0/).

\section{INFORMACIÓN DEL ARTÍCULO}

Historia del artículo:

Fecha recibido: junio 23 de 2020 Fecha aceptado: junio 26 de 2020

\footnotetext{
Autor para correspondencia.

Jorge Enrique Díaz Pinzón jediazp@unal.edu.co
}

DOI

10.31260/RepertMedCir.01217372.1082 


\section{A BSTRACT}

Introduction: after one hundred days of detecting the first case of Coronavirus disease 2019 (COVID-19) in Colombia, a research study was carried out regarding the geographic distribution of COVID-19 cases by departments and districts in the country. Objective: to compare if there are differences in the averages of positive cases, deaths and recoveries by departments and districts in Colombia. Materials and Methods: the database including people infected with COVID-19 was used, with the information collected as of June 13 2020. The T Student test was used for the statistical analysis of a sample in the SPSS v 25 statistical package. Results: the p-value obtained for positive cases is equal to 0.492 , for recoveries equal to 0.897 , and for deaths equal to 0.819 , in all three cases the p-value is greater than $a=0.05$, thus, the hypothesis (Ho) is accepted, indicating the $\mathrm{T}$ Student test applied to a sample resulted in no significant differences between the score for the population and the score obtained for the COVID-19 sample. Conclusions: the Andean zone of Colombia has a higher number of infected people, due to high population density, whereas, it is the opposite across the Amazon and Orinoquia regions. In addition, the same ratios are maintained for geographical regions in terms of recoveries and deaths.

Key words: coronavirus, infectious disease transmission; pandemic

(C) 2020 Fundación Universitaria de Ciencias de la Salud - FUCS. This is an open access article under the CC BY-NC-ND license (http://creativecommons.org/licenses/by-nc-nd/4.0/).

IN T RODUCCIÓN

Al cumplirse cien días del primer caso positivo del COVID-19 en Colombia, es relevante presentar un estudio de investigación del comportamiento de transmisión del coronavirus en la distribución geográfica en nuestro país. De acuerdo con Organización Mundial de la Salud. ${ }^{1,}$ en corto tiempo un brote delimitado de COVID-19 se convirtió en una pandemia mundial con tres características definitorias: rapidez y escala: la enfermedad se ha extendido ligeramente por todos los rincones del mundo y su velocidad de transmisión asombrosa ha rebosado incluso los sistemas sanitarios más resilientes.

Gravedad: $20 \%$ de los casos son graves o críticos, con una tasa de letalidad bruta de los casos clínicos que en este momento resalta el 3\%, y que es aún mayor en grupos de edad avanzada y en aquellas personas con ciertas enfermedades de base.

Perturbación social y económica: las perturbaciones a los sistemas sanitarios y de asistencia social y las medidas tomadas para vigilar la transmisión han tenido grandes y profundas consecuencias socioeconómicas. ${ }^{1,2}$

Por lo tanto, es necesario que instituciones como la ONU para la salud en las regiones, envíe más suministros vitales a los países más necesitados. ${ }^{3}$ Ahora mirando la situación en América Latina el panorama es mucho más oscuro. El coronavirus tardó en ingresar a la región, pero ahora está produciendo desgracias en muchos países y no se sabe cuánto más lejos consigue llegar. ${ }^{4}$

Estudios de investigación realizados por Díaz, ${ }^{5,6}$ el panorama no es nada halagador para Colombia, donde recomienda continuar con el aislamiento para que se genere una estabilización de la enfermedad; basado según cálculos de predicción del COVID-19 al mes de agosto 2020 habrá una cifra cercana a 70.000 contagios.

El objetivo de esta investigación es comparar si hay diferencias en los promedios de personas con contagio positivo, fallecidos y recuperados entre los diferentes departamentos y distritos de Colombia.

\section{METODOLOGÍA}

El trabajo de investigación se realizó mediante un enfoque cuantitativo según Hernández ${ }^{7}$ en un proceso secuencial y probatorio; se miden las variables en un establecido contexto; se analizan las mediciones alcanzadas (con frecuencia empleándose métodos estadísticos) y se instaura una serie de conclusiones con respecto a las hipótesis. Todos los experimentos cuantitativos utilizan un formato estándar con algunas pequeñas diferencias interdisciplinarias para generar una suposición que será probada o desmentida. Esta debe ser demostrable por medios matemáticos y estadísticos y constituye la base alrededor de la cual se diseña todo el experimento. ${ }^{8}$

\section{POBLACIÓN}

Esta investigación se centró en 37 departamentos y distritos especiales de Colombia, con la información del Instituto Nacional de Salud ${ }^{9}$ con corte a 13 de junio 2020 tabla 1. 
Tabla 1. Número de casos positivos, recuperados y fallecidos por COVID-19 por departamento y distritos. Colombia a 13 de junio 2020

\begin{tabular}{|c|c|c|c|}
\hline Departamentos y Distritos & Casos positivos & Recuperados & Fallecidos \\
\hline Nacional & 48756 & 19426 & 1592 \\
\hline Bogotá & 15458 & 6742 & 365 \\
\hline Barranquilla & 4862 & 1343 & 227 \\
\hline Valle del Cauca & 4690 & 1858 & 199 \\
\hline Cartagena & 4564 & 1473 & 187 \\
\hline Atlántico & 4215 & 1364 & 139 \\
\hline Amazonas & 2111 & 1232 & 77 \\
\hline Nariño & 2081 & 710 & 73 \\
\hline Antioquia & 1884 & 765 & 8 \\
\hline Cundinamarca & 1657 & 491 & 40 \\
\hline Meta & 1011 & 939 & 11 \\
\hline Buenaventura & 943 & 214 & 52 \\
\hline Choco & 680 & 137 & 17 \\
\hline Cesar & 528 & 73 & 13 \\
\hline Santa Marta & 480 & 247 & 24 \\
\hline Magdalena & 440 & 186 & 31 \\
\hline Tolima & 382 & 208 & 11 \\
\hline Bolívar & 341 & 140 & 17 \\
\hline Risaralda & 315 & 234 & 11 \\
\hline Huila & 272 & 232 & 9 \\
\hline Córdoba & 253 & 78 & 13 \\
\hline Boyacá & 241 & 150 & 9 \\
\hline Santander & 222 & 54 & 9 \\
\hline Sucre & 213 & 7 & 7 \\
\hline Caldas & 191 & 130 & 7 \\
\hline Cauca & 178 & 73 & 8 \\
\hline N Santander & 156 & 110 & 13 \\
\hline Quindío & 125 & 100 & 4 \\
\hline La Guajira & 121 & 48 & 7 \\
\hline Casanare & 37 & 33 & 0 \\
\hline Vaupés & 27 & 11 & 0 \\
\hline Caquetá & 25 & 21 & 1 \\
\hline San Andrés & 18 & 17 & 0 \\
\hline Putumayo & 13 & 4 & 2 \\
\hline Guainía & 7 & 0 & 1 \\
\hline Guaviare & 2 & 0 & 0 \\
\hline Arauca & 2 & 1 & 0 \\
\hline Vichada & 1 & 1 & 0 \\
\hline
\end{tabular}

Fuente: el autor.

Tabla 2. Estadísticas para una muestra

\begin{tabular}{|l|c|c|c|c|}
\hline Casos positivos & 37 & 1317,46 & 2778,374 & 456,762 \\
\hline Recuperados & 37 & 525,03 & 1162,952 & 191,188 \\
\hline Fallecidos & 37 & 43,03 & 79,797 & 13,119 \\
\hline
\end{tabular}

\section{ANÁLISIS ESTADÍSTICO}

Se plantearon hipótesis para la utilización de una prueba t student para las variables casos positivos, recuperados y fallecidos, diseñándose como una relación causal; se expone de la siguiente forma:
Prueba T para una muestra: El procedimiento prueba $\mathrm{T}$ para una muestra contrasta si la media de una sola variable difiere de una constante especificada. Consideraciones sobre los datos: para contrastar los valores de una variable cuantitativa con un valor de contraste hipotetizado se elige una variable cuantitativa y se introduce un valor de contraste hipotetizado. ${ }^{10}$

El test de la $\mathrm{t}$ de Student para una muestra admite comprobar si es posible aceptar que la media de la población es un valor señalado. Se toma una muestra y el test admite evaluar si es sensato sostener la hipótesis nula de que la media es tal valor. ${ }^{11}$

Hipótesis alternas (Ha): el valor de la media es diferente al valor de 1.000 en los casos positivos, el valor de la media es diferente al valor de 500 en los recuperados y el valor de la media es diferente al valor de 40 en los fallecidos entre los diferentes departamentos y distritos especiales de Colombia.

Hipótesis nula (Ho): el valor de la media es igual al valor de 1.000 en los casos positivos, el valor de la media es igual al valor de 500 en los recuperados y el valor de la media es igual al valor de 40 en los fallecidos entre los diferentes departamentos y distritos especiales de Colombia.

Prueba estadística: el valor de significancia de la prueba es de $a=0.05(5 \%)$, si es mayor se acepta la hipótesis nula si es menor se rechaza la hipótesis nula. Se realizó una comparación múltiple entre medias independientes, a partir del análisis estadístico de los datos aplicando la prueba $\mathrm{t}$ student para una muestra, se utilizó el paquete estadístico SPSS v. 25.

\section{RESULTADOS}

En la tabla 2 se observan las estadísticas para una muestra, en ella se describen las medias para casos positivos que es de 1317,46; 525,03 para recuperados, y 43,03 para fallecidos, para los diferentes departamentos y distritos de Colombia, con una población de 37.

En la tabla 3 se aprecian los diferentes p-valor de las pruebas, para la variable casos positivos es igual a 0,492, para la variable casos recuperados es igual a 0,897, para la variable fallecidos es igual a 0,819 , en los tres casos es mayor a $a=0.05$, de esta manera se acepta Ho, es decir la prueba t student para una muestra nos indica que no hay diferencias significativas entre el puntaje de la población y el obtenido para la muestra por COVID-19 para los diferentes departamentos y distritos de Colombia, con tres números de casos, contagio positivo $(\mathrm{M}=1317,46 ; \mathrm{t}=, 695 ; \mathrm{gl}=36 ; \mathrm{p}>$ $0,05)$; fallecidos $(M=525,03 ; \mathrm{t}=, 131 ; \mathrm{gl}=36 ; \mathrm{p}>0,05) ; \mathrm{y}$ recuperados $(\mathrm{M}=43,03 ; \mathrm{t}=, 231 ; \mathrm{gl}=36 ; \mathrm{p}>0,05)$.

En la figura 1 se observan los departamentos y distritos de Colombia para casos confirmados con fecha de corte 13 de junio 2020, tomado de la página del Ministerio de Salud y Protección Social ${ }^{12}$, se puede inferir que la zona con mayor 
de la región atlántica, continuando con la región pacífica, la región amazónica y finalmente con el menor número de casos confirmados la región Orinoquía.

En la figura 2 se aprecian los departamentos y distritos de Colombia para personas recuperadas, con fecha de corte 13 de junio 2020. Se puede analizar que los departamentos y distritos con mayor número de personas recuperadas son Bogotá tiene con 6742, seguida de Barranquilla con 1.343, Valle del Cauca con 1.858, Cartagena 1.473, Atlántico 1.364. Los departamentos y distritos con menor número de personas recuperadas son Vaupés 11, Sucre con 7, Putumayo 4, Guaviare y Guainía 0, Arauca y Vichada 1.

Tabla 3. Prueba para una muestra

\section{Valor de prueba $C P=1000$, recuperados $=500$, fallecidos $=40$}

\begin{tabular}{|c|c|c|c|c|c|c|}
\hline & \multirow[b]{2}{*}{$t$} & \multirow[b]{2}{*}{ gl } & \multirow{2}{*}{$\begin{array}{c}\text { Significancia. } \\
\text { (bilateral) }\end{array}$} & \multirow{2}{*}{$\begin{array}{l}\text { Diferencia de } \\
\text { medias }\end{array}$} & \multicolumn{2}{|c|}{$95 \%$ de intervalo de confianza de la diferencia } \\
\hline & & & & & Inferior & Superior \\
\hline Casos positivos & ,695 & 36 & 492 & 317,459 & $-608,90$ & 1243,82 \\
\hline Recuperados &, 131 & 36 & 897 & 25,027 & $-362,72$ & 412,77 \\
\hline Fallecidos & ,231 & 36 & ,819 & 3,027 & $-23,58$ & 29,63 \\
\hline
\end{tabular}
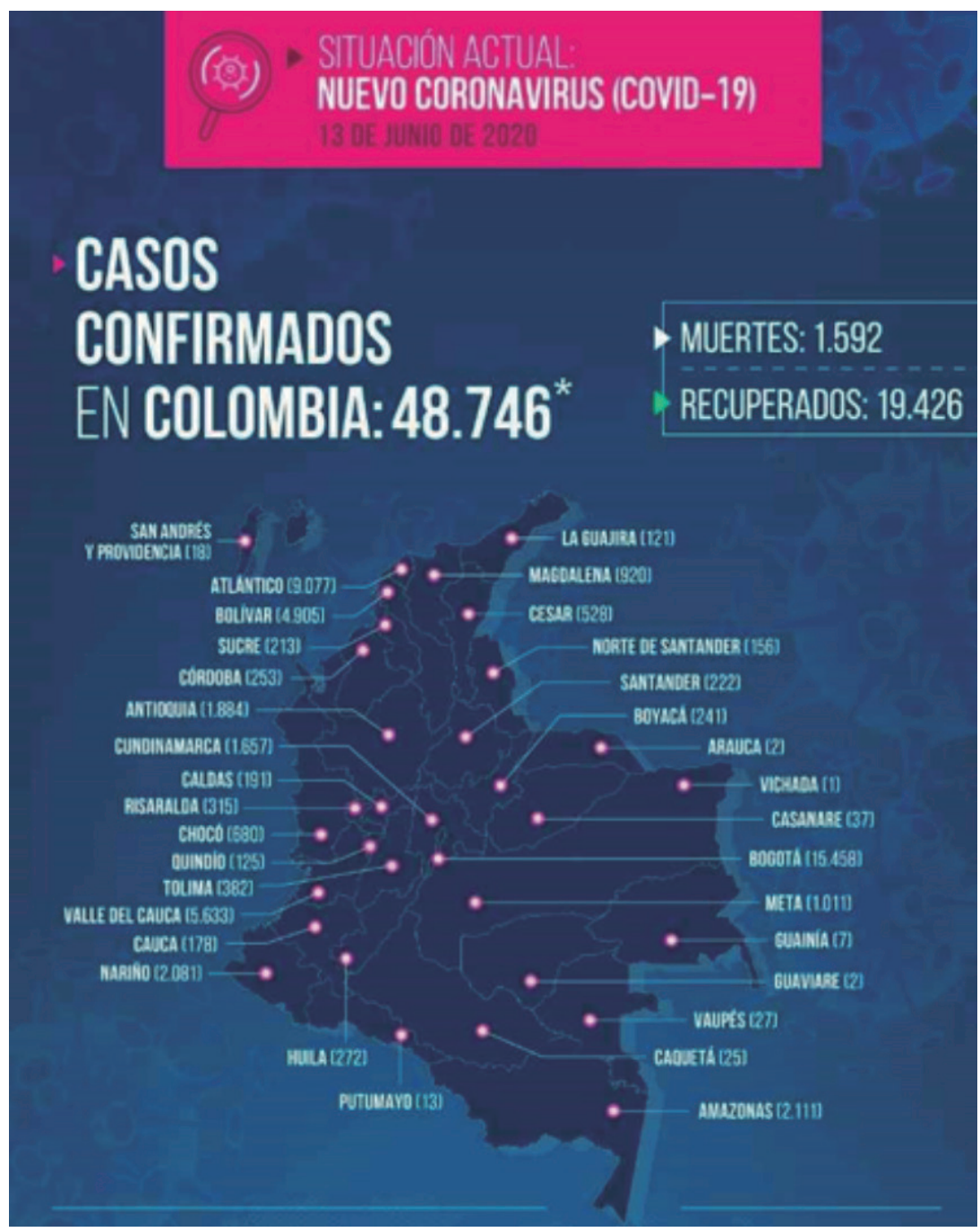

Figura 1 . Casos confirmados en Colombia a 13 de junio 2020. Fuente: Ministerio de Salud y Protección Social. ${ }^{12}$ 
En la figura 3 se aprecian los departamentos y distritos de Colombia para personas fallecidas, con fecha de corte 13 de junio 2020. Se puede analizar que los departamentos y distritos con mayor número de personas fallecidas son Bogotá 365, seguida de Barranquilla 227, Valle del Cauca 199, Cartagena 187, Atlántico 139; y los departamentos y distritos con menor número de personas recuperadas son: Vaupés 0, San Andrés 1, Putumayo 0, Guaviare y Guainía 0, Arauca y Vichada 0. Este aumento en los resultados es debido al contagio de personas con la apertura global y sistemática de varios sectores de la economía de acuerdo con Díaz. ${ }^{13}$ Se debe continuar con los protocolos de bioseguridad para todos los grupos poblacionales (niños y adolescentes, adultos mayores de 70 años, personas con preexistencias médicas, grupos étnicos) y para todos los sectores en proceso de rescatar vida productiva: servicios, construcción, manufactura, comercio, bancos, agropecuario y educación, deporte y cultura, entre otros. ${ }^{14}$

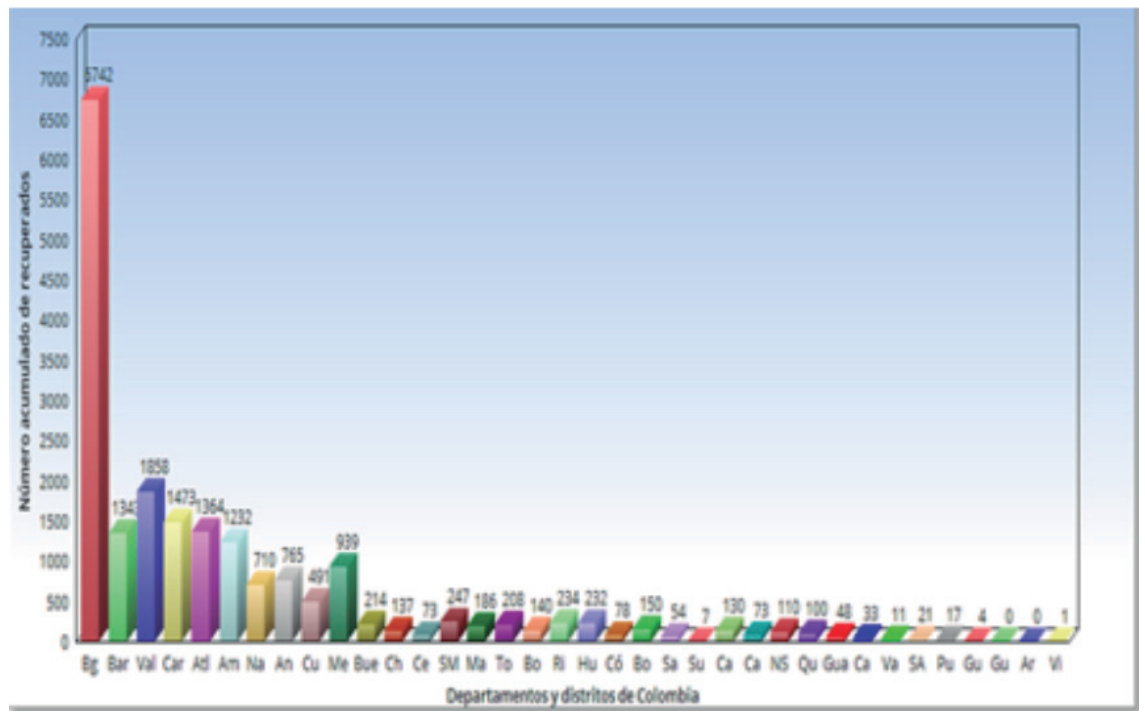

Figura 2. Recuperados en Colombia a 13 de junio 2020. Fuente: el autor.

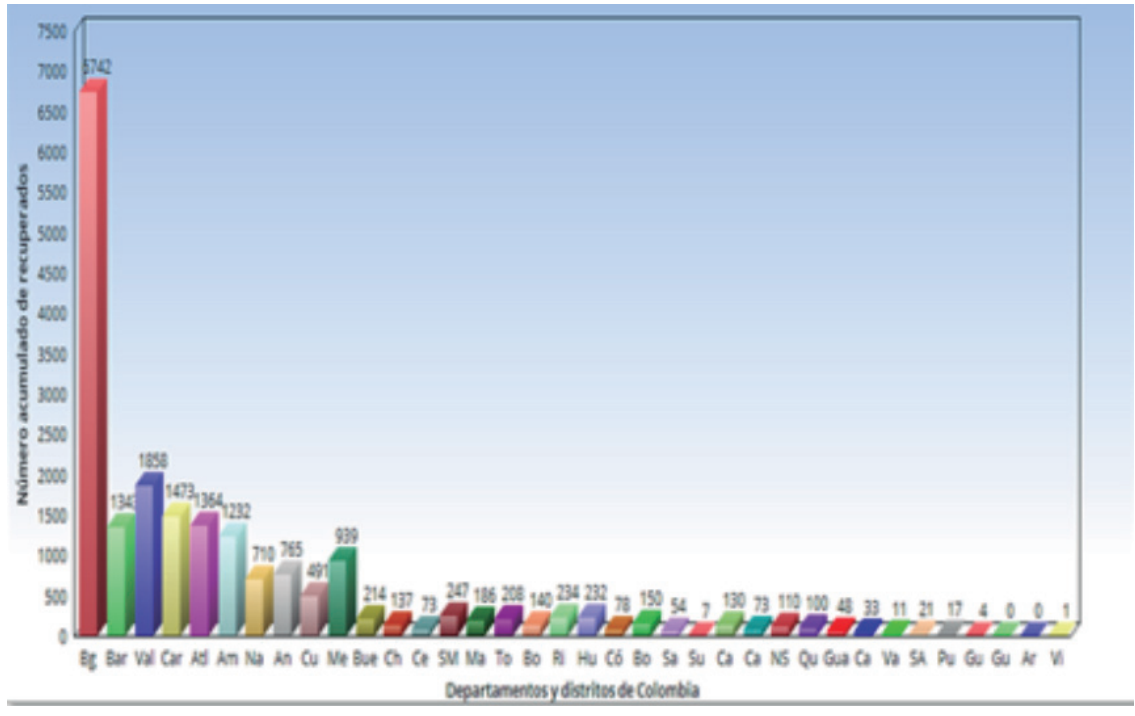

Figura 3. Fallecidos en Colombia a 13 de junio 2020. Fuente: el autor. 


\section{CONCLUSIONES}

A partir del análisis estadístico de los datos aplicando la T-Student para una muestra, los diferentes p-valor de las pruebas para la variable casos positivos es igual a 0,492, para la variable casos recuperados es igual a 0,897, para la variable fallecidos es igual a 0,819 , en los tres casos es mayor a $a=0.05$, de esta manera se acepta Ho, es decir la prueba t student para una muestra nos indica que no hay diferencias significativas entre el puntaje de la población y el obtenido para la muestra por COVID-19 para los diferentes departamentos y distritos de Colombia, a fecha de corte 13 de junio 2020.

De acuerdo con el promedio nacional de personas con casos positivos que fue de 1317,46, los departamentos y distritos de Colombia que están por encima de este referente son en su orden Bogotá (1545.8), Barranquilla (4.862), Valle del Cauca (4.690), Cartagena (4.564), Atlántico (4.215), Amazonas (2.111), Nariño (2.081), Antioquía (1.884), y Cundinamarca (1.657).

Acorde con el promedio nacional de personas recuperadas que fue de 525,03, los departamentos y distritos de Colombia que están por encima de este referente son en su orden Bogotá (6742), Barranquilla (1.343), Valle del Cauca (1.858), Cartagena (1.473), Atlántico (1.364), Amazonas (1.232), Antioquía (765) y Nariño (710).

Acorde con el promedio nacional de personas fallecidas que fue de 43,03, los departamentos y distritos de Colombia que están por encima de este referente son en su orden Bogotá (365), Barranquilla (227), Valle del Cauca (199), Cartagena (187), Atlántico (139), Amazonas (77), Nariño (73) y Buenaventura (52).

Se presenta mayor población de contagio en la zona Andina de Colombia debido a la mayor densidad de población en esta región, y lo contrario en las regiones de la Amazonía y Orinoquía. Además, se mantiene la misma relación por zonas geográficas en cuanto a personas recuperadas y fallecidas.

\section{REFERENCIAS}

1. Organización Mundial de la Salud. OMS. Actualización de la estrategia frente a la covid 19 [Internet]. 2020 [citado 2020 junio 10]. Recuperado de: https://www.who.int/docs/defaultsource/coronaviruse/covid-strategy-update-14april2020_ es.pdf?sfvrsn=86c0929d_10

2. Organización Internacional del Trabajo. OIT. La COVID-19 y el mundo del trabajo [Internet]. 2020 [citado 2020 junio 10]. Recuperado de: https://www.ilo.org/global/topics/coronavirus/lang--es/ index.htm

3. Naciones Unidas. La enfermedad del coronavirus, una emergencia de salud mundial [Internet]. 2020 [citado 2020 junio 10]. Recuperado de: https://www.un.org/es/coronavirus
4. Infobae. Los nueve países que derrotaron al coronavirus [Internet]. 2020 [citado 2020 junio 10]. Recuperado de: https://www. infobae.com/america/mundo/2020/06/09/los-nueves-paises-quederrotaron-al-coronavirus/

5. Díaz Pinzón, J.E. Uso de modelo predictivo para la dinámica de transmisión del COVID-19 en Colombia. Repert. Med. Cir. 2020;29(Núm. Supl.1):34-44

6. Díaz Pinzón, J.E. Precisión del pronóstico de la propagación del COVID-19 en Colombia. Repert. Med. Cir. 2020;29(Núm. Supl.1):27-33

7. Hernández, R. Fernández, C. Baptista, M. Metodología de la Investigación [Internet]. 7 de mayo de 2020 [citado 11 de junio de 2020]; Disponible en: https://www.esup.edu.pe/ descargas/dep_investigacion/Metodologia \%20de \% 20la \% 20 investigaci\%C3\%B3n \%205ta\%20Edici\%C3\%B3n.pdf

8. Shuttleworth, M. Diseño de la investigación cuantitativa [Internet]. 2008 [citado 2020 junio 11]. Recuperado de: https://explorable. com/es/diseño-de-lainvestigación-cuantitativa

9. Instituto Nacional de Salud de Colombia. Coronavirus (CO-VID-19) en Colombia [Internet]. 2020 [Citado 30 de mayo de 2020]. Disponible en: https://bit.ly/2UNnOtl

10. IBM Knowledge Center. Prueba $\mathrm{T}$ para una muestra [Internet]. 2020 [citado 2020 junio 15]. Disponible en: https://www.ibm. com/support/knowledgecenter/es/SSLVMB_sub/statistics_ mainhelp_ddita/spss/base/idh_ttss.html

11. Llopis, Jaume. Test de la t de Student para una muestra [Internet]. 2020 [citado 2020 junio 15]. Disponible en: https://jllopisperez. com/2012/12/16/test-de-la-t-de-student-para-una-muestra/

12. Ministerio de Salud y Protección Social. Conozca toda la información relacionada con el coronavirus (COVID-19). [Internet]. 2020 [citado 2020 junio 15]. Disponible en: https:// d2jsqrio60m94k.cloudfront.net/

13. Díaz Pinzón, J.E. Estudio comparativo entre el contagio durante la cuarentena obligada por el COVID-19 y el contagio durante la apertura gradual y controlada para algunos sectores de la economía en Colombia. Repert. Med. Cir. 2020;29 (Núm. Supl.1):52-58. doi: 10.31260/RepertMedCir.01217372.1073

14. Presidencia. Dos meses de cuarentena / Colombia tiene hoy un sistema de salud más fortalecido y preparado para salvar vidas. [Internet]. 2020 [citado 25 de mayo de 2020]; Disponible en: https://bit.ly/36Bussk 